\title{
EIXOS MOBILIZADORES EM QUÍMICA
}

Jailson B. de Andrade*

Instituto de Química, Universidade Federal da Bahia, 40170-290 Salvador - BA

Solange Cadore

Instituto de Química, Universidade Estadual de Campinas, CP 6154, 13084-971 Campinas - SP

Paulo C. Vieira

Departamento de Química, Universidade Federal de São Carlos, CP 676, 13565-905 São Carlos - SP

César Zucco

Departamento de Química, Universidade Federal de Santa Catarina, CP 476, 88040-900 Florianópolis - SC

Ângelo C. Pinto

Instituto de Química, Universidade Federal do Rio de Janeiro, CT, Bloco A, Cidade Universitária, Ilha do Fundão, 21949-900 Rio de Janeiro - RJ

\begin{abstract}
MOBILIZING AXES IN CHEMISTRY. Over the last five years there have been significant changes in higher education in Brazil as well as in research funding. As a contribution to the development of Science and Technology, and aiming to portray Chemistry today in Brazil, in the context of last year's elections for President, State Governors, National Congress and Legislative Chamber, the Directors and Consulting Council of the Brazilian Chemical Society, SBQ, initiated in 2002 a series of activities to produce a document entitled Mobilizing Axes in Chemistry. This discusses undergraduate and graduate teaching in Chemistry, a new model for research funding, and the overall state of the art, and future perspectives. Six mobilizing axes have been identified and discussed to date: 1 . Training of highly qualified personnel; 2. Decentralization, and discouragement of institutional in-breeding; 3. Stimulation of entrepreneurship and interdisciplinarity; 4. A guaranteed budget for Science and Technology; 5. Proactive interaction of academics with economic activity; and 6. Removal of institutional bottle-necks of all sorts. The Brazilian Chemical Society hopes that the new administration will in the near future begin the task of improving the national education system and increase funding for Science and Technology.
\end{abstract}

\section{MOTIVAÇÃO}

Nos últimos cinco anos ocorreram mudanças significativas no sistema de ensino superior brasileiro e no financiamento à pesquisa. Foram criadas as Diretrizes Curriculares para os cursos de graduação, e o sistema de avaliação dos cursos de pós-graduação, feito pela CAPES, foi alterado, com a ampliação do elenco de conceitos (introdução das notas 6 e 7) e a criação do sistema de classificação de periódicos denominado QUALIS. Por outro lado, o modelo de financiamento da pesquisa científica e tecnológica pelo Governo Federal foi totalmente reformulado, tendo sido introduzidos os Fundos Setoriais, os Institutos do Milênio, os Programas Temáticos e os Editais Universais do CNPq. Neste novo cenário, o denominado "balcão" das Agências Federais foi praticamente substituído pelo Edital Universal do CNPq em 2000 e 2001 (em dezembro de 2002 foi lançado o Edital 01/2002 para financiamento de projetos a partir de junho de 2003). Nestes, a demanda da área de Química superou significativamente o volume total de recursos disponíveis e, mesmo com uma demanda maior do que todos os recursos disponíveis nos Editais, após a avaliação, foram destinados para a Química, em 2000 e 2001, respectivamente, $6,1 \%$ e $4,0 \%$ do total de recursos.

A área de Química é uma das que apresenta maiores taxas de crescimento na formação de mestres e doutores, bem como no número de publicações de artigos em periódicos científicos de reconhecida qualidade. Entretanto, o acesso ao financiamento com re-

*e-mail: jailsong@ufba.br cursos oriundos do Governo Federal encontra-se aquém das necessidades e do potencial da área. Com o objetivo de contribuir para a formulação de políticas de C\&T para a área de Química, a Diretoria e o Conselho Consultivo da Sociedade Brasileira de Química, SBQ, decidiram em 2002 promover uma série de atividades visando a elaboração de um documento denominado "Eixos Mobilizadores em Química" e a discussão de temas como o ensino de graduação e pósgraduação em Química, o novo modelo de financiamento à pesquisa, a situação da área e suas perspectivas, especialmente considerando as eleições para Presidente, Governadores, Congresso Nacional e Assembléias Legislativas em 2002 e as respectivas posses em 2003.

$\mathrm{Na} 25^{\text {a }}$ Reunião Anual da SBQ, em Poços de Caldas, MG, em maio de 2002, foi realizado o workshop "UM OLHAR SOBRE A PÓS-GRADUAÇÃO EM QUÍMICA NO BRASIL”, coordenado pelos Conselheiros Solange Cadore (UNICAMP) e Jailson B. de Andrade (UFBA), que contou com a presença, entre outros, de representantes da CAPES (Luiz V. Loureiro) e do CNPq (Celso P. Melo), do Coordenador da área de Química na CAPES e Membros do Comitê de Avaliação, além de Coordenadores de cursos de Pós-Graduação em Química (Quadro 1). Outra atividade realizada foi o Simpósio noturno, "EIXOS MOBILIZADORES EM QUÍMICA", contando com a participação de Eliezer J. Barreiro (Presidente da SBQ no biênio 2000-2002), Eduardo M. Krieger (Presidente da Academia Brasileira de Ciências) e Jailson B. de Andrade (UFBA). O workshop "A QUÍMICA NO BRASIL: SITUAÇÃO ATUAL E PERSPECTIVAS”, coordenado pelos Conselheiros Solange Cadore (UNICAMP) e Jailson B. de Andrade (UFBA), ocorreu em Salvador, BA, de 04 a 05 de dezembro de 2002, com o objetivo principal de dar continuidade às discussões sobre o ensino, pesquisa e avaliação em Química, com a participação dos (as) Coordenadores (as) de Programas de Pós-gradua- 
ção em Química, Diretoria e Conselho da SBQ, Membros dos Comitês Assessores de Química na CAPES, CNPq e Fundações Estaduais de Fomento (Quadro 2). A última etapa desta série de eventos será o Simpósio “A FORMAÇÃO DO QUÍMICO” que ocorrerá na 26a Reunião Anual da SBQ, em maio de 2003.

\section{O CONHECIMENTO}

A humanidade esteve envolvida em duas grandes ondas de transformação no passado: Agricultura e Industrialização. No momento, está envolvida em duas outras grandes ondas: Comunicação e Conhecimento. O conhecimento está intimamente relacionado com três vertentes: Geração, Difusão e Apropriação. Esta última vertente manifesta-se com uma forte simbiose com a Proteção do conhecimento. O conhecimento e suas vertentes têm como alvo o setor em- presarial, o governo, as organizações não governamentais e a sociedade em geral (Figura 1).

A relação entre a geração do conhecimento e a difusão é uma linha reta. Entretanto, a relação entre geração do conhecimento, sua apropriação e inovação tecnológica não o é (Figura 2). A geração do conhecimento está associada à pesquisa básica, que envolve a formação de cientistas e fornece as sementes do conhecimento para o interesse futuro da indústria. Geralmente requer investimentos de longo prazo - pelo menos 10 anos - e o principal financiador é o Governo Federal. Enquanto a pesquisa básica busca a compreensão de fenômenos, a tecnológica busca a utilização prática do conhecimento $^{1}$ e está intimamente relacionada com a inovação tecnológica e a apropriação do conhecimento. Assim, a pesquisa tecnológica requer resultados de curto prazo - 3 a 5 anos - e recebe, geralmente, investimento do setor privado (Figura 3).

Quadro 1. Programa do workshop “Um Olhar sobre a Pós-graduação em Química no Brasil”, Poços de Caldas, MG, 20 de maio de 2002

\begin{tabular}{lll}
\hline HORÁRIO & ATIVIDADE & CONFERENCISTA \\
\hline 09:20 - 09:30 & Abertura & \\
09:30 - 10:20 & A Evolução da Pós-graduação em Química no País & Jailson B. de Andrade (UFBA) \\
10:40 - 12:00 & A Expansão e o Financiamento para a Pós-graduação & Celso P. Melo (CNPq), Luiz V. Loureiro (CAPES) \\
14:00 - 15:00 & O sistema de avaliação da Pós-Graduação & Alfredo Arnóbio Gama (UFPe) e Vitor F. Ferreira (UFF) \\
15:00 - 16:00 & Perspectivas para a Pós-graduação & Ângelo C. Pinto (UFRJ) \\
16:00 - 17:00 & Plenária & \\
17:00 & Encerramento & \\
\hline
\end{tabular}

Quadro 2. Programa do workshop "A Química no Brasil: Situação Atual e Perspectivas”, Salvador, BA, 04-05 de dezembro de 2002

\begin{tabular}{|c|c|}
\hline Horário & $04 / 12 / 2002$ \\
\hline 09:00h - 09:30h & $\begin{array}{l}\text { Abertura: Paulo C. Vieira (Presidente da SBQ); Frederico G. Cruz (Diretor do IQ-UFBA), Solange Cadore (Coordenação } \\
\text { do WS) }\end{array}$ \\
\hline 09:30h - 10:00h & Jailson B. de Andrade (UFBA-SBQ), "Eixos Mobilizadores em Química" \\
\hline $10: 00 \mathrm{~h}-10: 30 \mathrm{~h}$ & Alfredo Arnóbio Gama (UFPe-CAPES), "O Papel da CAPES na avaliação da PG e no cenário de C\&T” \\
\hline $10: 30 h-11: 00 h$ & Cezar Zucco (UFSC-CNPq), "O papel do CNPq no Financiamento de C\&T” \\
\hline $11: 00 \mathrm{~h}-11: 30 \mathrm{~h}$ & Ângelo C. Pinto (UFRJ), "Perspectivas para a Pesquisa e Pós-graduação" \\
\hline $11: 30 \mathrm{~h}-12: 00 \mathrm{~h}$ & Mauro M. Braga (UFMG), "O Destino de Mestres e Doutores em Química” \\
\hline $12: 00 h-12: 45 h$ & Sessão Plenária \\
\hline \multirow[t]{8}{*}{$14: 45 h-16: 30 h$} & Trabalho em Grupo: \\
\hline & $\begin{array}{l}\text { Grupo } 1 \text { - Avaliação: do Pesquisador, Grupo de Pesquisa e da Pós-graduação, Coordenador - Alfredo Arnóbio Gama; } \\
\text { Relator - Claudia M. de Rezende; }\end{array}$ \\
\hline & $\begin{array}{l}\text { Grupo } 2 \text { - Programas recentes: PROCAD, PQI, PROFIX e PRONEX, Coordenador - Vitor F. Ferreira; Relator - } \\
\text { Solange Cadore; }\end{array}$ \\
\hline & $\begin{array}{l}\text { Grupo } 3 \text { - Formação de Recursos Humanos: da graduação à Pós-graduação, Coordenador - Cezar Zucco; Relator - } \\
\text { Antonio S. Mangrich; }\end{array}$ \\
\hline & Grupo 4 - Desconcentração Regional e Combate a Endogenia, Coordenador - Oswaldo L. Alves; Relator - Mara E. F. \\
\hline & Braibante; \\
\hline & $\begin{array}{l}\text { Grupo } 5 \text { - Gargalos Institucionais e Interdisciplinaridade, Coordenador - Frederico G. Cruz; Relator - Timothy J. } \\
\text { Brocksom }\end{array}$ \\
\hline & Grupo 6 - Financiamento da Pesquisa e Pós-Graduação; Coordenador - Fernando Galembeck; Relator - Faruk Nome; \\
\hline $16: 30 h-18: 30 h$ & Sessão Plenária \\
\hline Horário & $05 / 12 / 2002$ \\
\hline 08:30h - 10:00h & Trabalho em Grupo \\
\hline $10: 00 h-12: 30 h$ & Sessão Plenária - Eixos Mobilizadores em Química; Coordenador - Paulo C. Vieira; Relator - Jailson B. de Andrade \\
\hline $14: 00 h-16: 00 h$ & Sessão Plenária, Documento final, recomendações \\
\hline $16: 00 h-16: 30 h$ & Sessão de encerramento \\
\hline
\end{tabular}




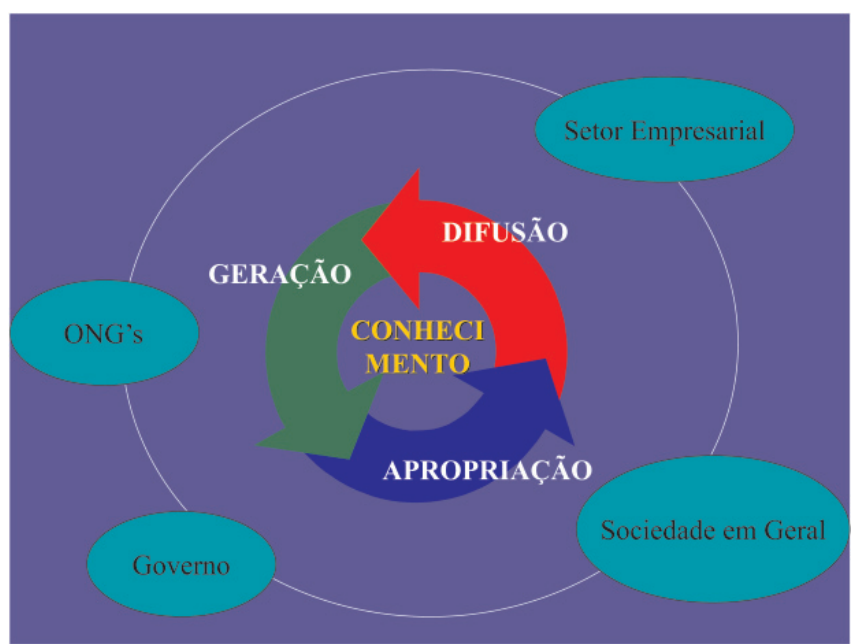

Figura 1. O conhecimento e as suas relações com a Geração, Difusão e Apropriação

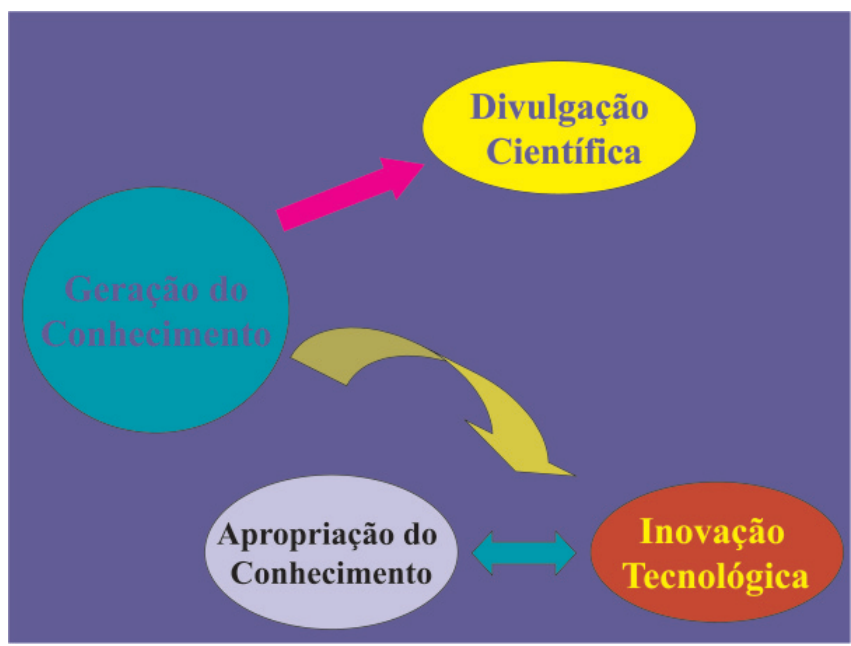

Figura 2. Relação entre geração do conhecimento, apropriação do conhecimento e inovação tecnológica

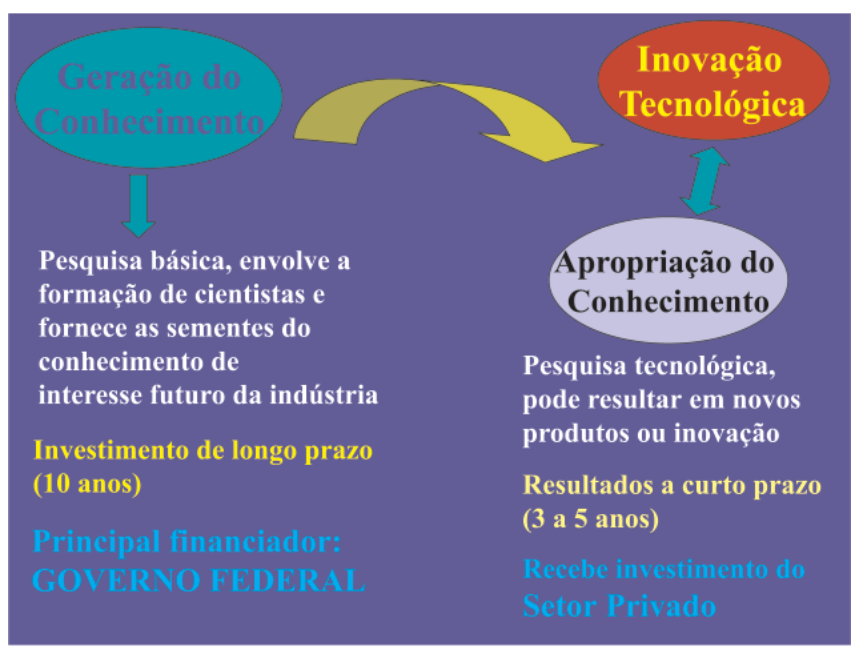

Figura 3. Inter-relações entre pesquisa básica e tecnológica

A apropriação do conhecimento tem sido objeto de discussão permanente entre cientistas e financiadores da pesquisa. Recentemente, Stokes publicou o livro "O Quadrante de Pasteur"2 em que apresenta um novo paradigma de relacionamento entre pesquisa básica e pesquisa aplicada, sugerindo que a ciência básica, no mundo pós-guerra, deve ser direcionada especificamente para o desenvolvimento de novos produtos ou aplicações. Nesse novo contexto, a ciência básica, dirigida simplesmente para a geração de conhecimento, não deveria ser financiada. O Quadrante de Pasteur (Figura 4) coloca em evidência o trabalho de Louis Pasteur como ciência básica, que resultou em aplicações. Este quadrante é contíguo ao Quadrante de Bohr (Niels Bohr), que representa essencialmente a pesquisa básica, e com o Quadrante de Edson (Thomas Edson), que representa a pesquisa essencialmente aplicada ou pesquisa em engenharia.

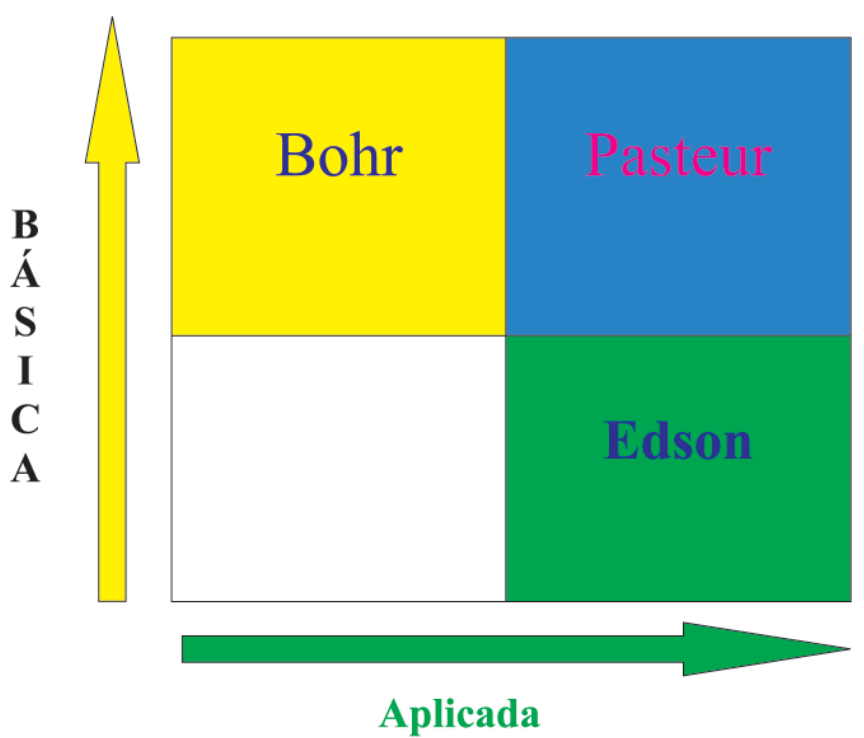

Figura 4. O Quadrante de Pasteur ${ }^{2}$

O relacionamento entre pesquisa básica e pesquisa aplicada tem se tornado bastante popular no mundo todo e, especialmente, no Brasil. Em 2001, o Ministério da Ciência e Tecnologia, MCT, incorporou uma nova letra I (de inovação), em suas atividades. Elaborou o livro verde de C,T\&I e organizou uma Conferência Nacional em Ciência, Tecnologia e Inovação, cujo subtítulo era Conhecimento $e$ Inovação para o Futuro. Uma comparação tem sido recorrente no sistema de C\&T: o Brasil e a Coréia do Sul estão entre os países de maior taxa de crescimento em publicações indexadas e representam 1,3-1,5\% das publicações indexadas no Institute for Scientific Information (ISI) ${ }^{3}$. Entretanto, quando comparados com relação a Patentes depositadas nos Estados Unidos, as do Brasil representam menos de $2 \%$ do total anual da Coréia ${ }^{4}$. Os motivos dessa discrepância são variados, e um grande destaque tem sido dado ao investimento de recursos pelo setor empresarial em pesquisa na Coréia - que é significativamente maior do que no Brasil - e ao número de cientistas e engenheiros engajados em pesquisa: no Brasil o maior percentual está na academia (68\%), enquanto que na Coréia o maior percentual está nas empresas (54\%). Essas comparações são relevantes! Entretanto, dois fatos importantes devem ser considerados: o número de cientistas e engenheiros envolvidos em pesquisa na Coréia é praticamente o dobro do existente no Brasil (Figura 5) e não existem analfabetos naquele país.

\section{FORMAÇÃO DE PESSOAL DE ALTO NÍVEL}

Essas e outras análises comparativas evidenciam que para o país dar um salto também em inovação é necessário um investimento sig- 


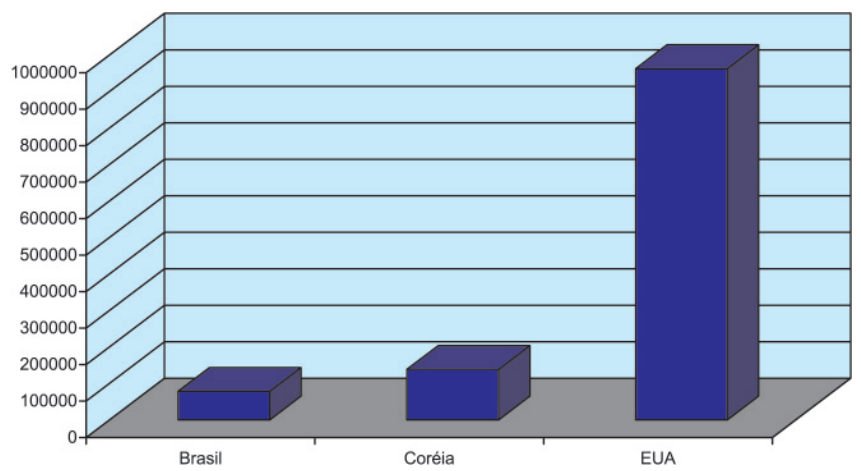

Figura 5. Número de cientistas e engenheiros envolvidos em pesquisa no Brasil, Coréia e Estados Unidos ${ }^{3}$

nificativo na formação de recursos humanos qualificados em profusão e em todos os níveis. Logo, o primeiro eixo mobilizador sugerido é a formação de recursos humanos qualificados. Associadas a este eixo estão algumas ações, tais como: estímulo ao acesso direto ao doutorado aos alunos que participaram do programa de iniciação científica; incentivo às atividades conjuntas da graduação e da pós-graduação; promoção de intercâmbio de Química entre as IES e também com o nível médio; o pós-doutorado deve ser considerado um processo de formação continuada, abrangendo estágios posteriores ao doutorado; constituição de um grupo de trabalho da SBQ para acompanhar a implantação das diretrizes curriculares nas IES; incentivo a programas para formação de docentes de $3^{\circ}$ grau; realização de Semanas Nacionais de Química com a participação de estudantes do nível médio, de graduação, pós-graduação e professores, como forma de mobilização e integração entre os vários níveis de ensino.

\section{PÓS-GRADUAÇÃO E PESQUISA}

Nos últimos anos e, principalmente, a partir de 1983, a pesquisa e a pós-graduação na área de Química mostraram um crescimento bastante acentuado, de tal forma que, hoje, o Brasil supera a contribuição conjunta de México, Chile e Argentina em relação à produção científica encontrada em publicações indexadas no ISI, ao faturamento da indústria química e ao número de cursos de doutorado em Química ${ }^{5-7}$. A avaliação dos cursos de pós-graduação em Química pela CAPES, referente ao período 1998-2000, registrou a existência, em 2000, de 1.203 alunos matriculados no mestrado e 1.471 no doutorado e a titulação de 384 mestres e 244 doutores. Naquele mesmo ano foram publicados 2.208 artigos, sendo 478 no país e 1.730 no exterior. Uma visão geral da qualidade dos cursos e dos artigos pode ser obtida pela média dos conceitos dos cursos que é cinco numa escala de um a sete, e no fator de impacto ${ }^{8}$ médio das publicações da área no Brasil $(1,03)$ e em todo o mundo $(1,32)^{9}$.

Atualmente, existem 42 cursos de mestrado e 29 cursos de doutorado em Química no país e, dos 15.158 grupos de pesquisa cadastrados no $\mathrm{CNPq}$, 685 são de Química. O conjunto de cursos de pós-graduação e de grupos de pesquisa é expressivo, uma vez que a área representa $4,5 \%$ dos grupos de pesquisa do país e é a terceira em tamanho. Entretanto, mais de $90 \%$ dos cursos de pós-graduação e dos grupos de pesquisa concentram-se nas regiões Nordeste, $\mathrm{Su}-$ deste e Sul com uma segunda concentração na região Sudeste que, sozinha, detém mais de $50 \%$ dos cursos e grupos de pesquisa (Figura 6). Por outro lado, o número de estudantes de doutorado ou pósdoutorado, com bolsas da CAPES e/ou CNPq realizando estudos no exterior é inexpressivo.

Dois desafios são de grande relevância nesse contexto: promover a desconcentração regional sem perda de qualidade e combater a

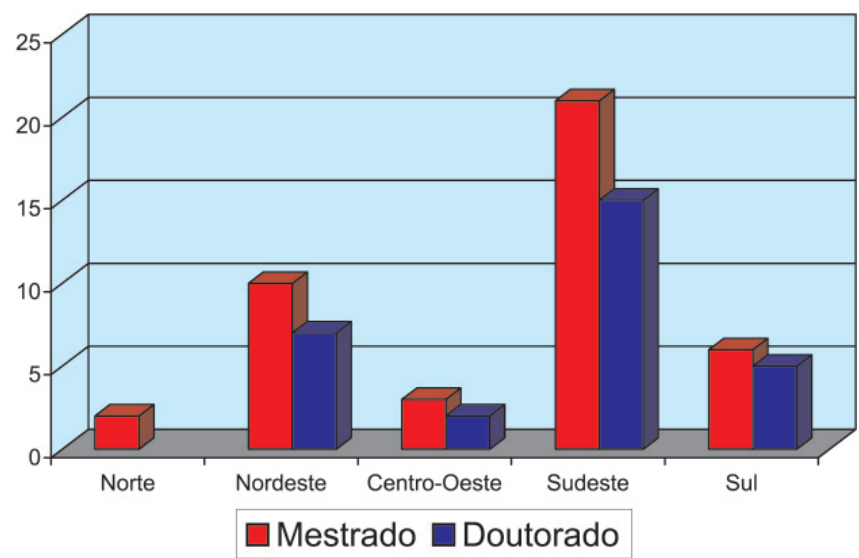

Figura 6. Distribuição regional dos cursos de mestrado e doutorado em Química

endogenia em qualquer das suas expressões - individual, temática ou geracional. Logo, o segundo eixo mobilizador sugerido é desconcentração regional e combate a endogenia. Algumas ações associadas a este eixo são: criação de facilidades regionais compartilhadas, para uso de equipamentos de médio e grande porte; acesso aos grandes centros, estabelecendo programas de intercâmbio (docentes, discentes, técnicos) visando a formação de recursos humanos; criação de programas regionais induzidos de fixação objetivando a capacitação de pessoal; financiamento diferenciado com características regionais, visando melhorar a capacidade laboratorial, computacional e de serviços de grupos de pesquisas existentes e em formação; utilização de recursos e facilidades da informática como forma de disseminação de informação (vídeocursos, vídeoconferências, operação remota de equipamentos); formação de núcleos de programas de pós-graduação e capacitação; simplificação do processo jurídico em situações que envolvem doação/transferência de equipamentos entre instituições e, a valorização de iniciativas que induzam à mobilidade de docentes, pesquisadores e discentes entre as IES do país (PROFIX, PRODOC e outros que possam vir a ser criados).

\section{QUÍMICA A PALAVRA ESCONDIDA}

Sem a Química seria impossível manter a qualidade de vida da humanidade com alimentos, segurança ambiental, longevidade e conforto. Entretanto, freqüentemente tem sido observada, erroneamente, a associação da palavra química com poluição, armas e drogas, além do crescente desuso das divisões didáticas originais da Química: Química Analítica, Química Inorgânica, Química Orgânica e Físico-Química. O exemplo mais recente foi o seminário "Pós-graduação Enfrentando Novos Desafios", em que um levantamento das quatro palavras mais freqüentes relacionadas à Química - representando quase 50\% do total de citações - indicou Ciência dos Materiais, Química Ambiental, Instrumentação e Fármacos (Figura 7), não incluindo, portanto, qualquer das divisões tradicionais da Química ${ }^{10}$.

Nessa mesma direção, Pearce, Presidente da American Chemical Society em 2002, na mensagem presidencial assim se manifestou ${ }^{11}$ : "There is no doubt in my mind that the traditional educational divisions of chemistry are historical artifacts ..." e, mais adiante "Speaking on this subject and considering the narrow breadth and width of current chemical education, I am reminded of Carl Djerassi's wonderful phrasing urging new science and Engineering graduates to practice 'intellectual pollygamy' and 'intellectual promiscuity' in this era of rampant overspecialization”. 


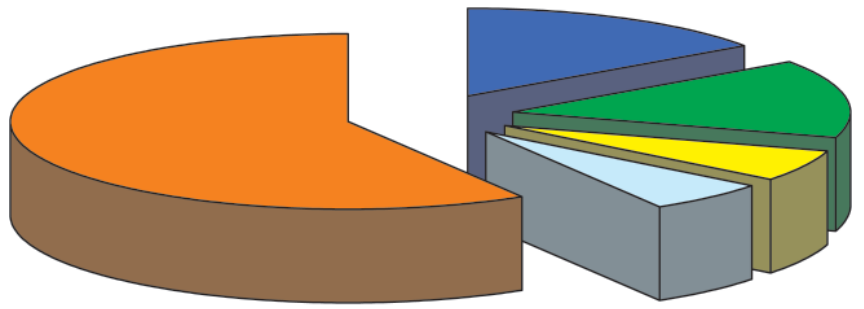

\begin{tabular}{|ll|}
\hline Ciência dos Materiais & $\square Q$. Ambiental \\
$\begin{array}{l}\square \text { Instrumentação } \\
\square \text { Outros }\end{array}$ & $\square$ Fármacos \\
\hline
\end{tabular}

Figura 7. Palavras mais freqüentes relacionadas à Química ${ }^{9}$

Uma das experiências significativas no financiamento à pesquisa é realizada na Austrália através do Commonwealth Scientific \& Industrial Research Organization - CSIRO (www.csiro.au) onde 21 estruturas de pesquisa são financiadas regularmente pelo governo. A presença da Química pode ser percebida facilmente em todas as estruturas. Entretanto, no título de nenhuma delas existe a palavra Química.

Neste contexto, se fosse possível uma reorganização da área em temáticas mais amplas, quatro seriam suficientes e englobariam as quatro palavras chaves mais citadas no seminário "Pós-graduação Enfrentando Novos Desafios": Química dos Materiais, Química do Ambiente, Química da Vida e Instrumentação.

Por outro lado, com relação à formação do graduado em Química, constata-se que os currículos vigentes na maioria das IES brasileiras formam químicos para o setor industrial e/ou estudantes de pós-graduação. Com a crescente escassez de emprego nas grandes empresas e com a implantação de novos modelos de desenvolvimento, torna-se urgente, também, dirigir a formação dos profissionais da Química no sentido de atribuir-lhes competência técnica para poder empreender o seu próprio negócio e/ou atuar de forma criativa em seu trabalho ${ }^{12}$. A absorção do pós-graduado em Química pelo setor industrial tem sido muito tímida, como mostra o trabalho realizado por Braga e Azevedo ${ }^{13}$. O levantamento feito com egressos da pós-graduação constatou que cerca de $25 \%$ dos mestres e somente 5\% dos doutores em Química são absorvidos pelas empresas. A grande maioria dos pós-graduados busca as instituições de ensino superior e centros de pesquisa após a obtenção do título. A pesquisa revela ainda que esta tendência não está associada ao salário pago a esses profissionais. Por outro lado, foi detectado também que as empresas começaram a perceber a importância de contratar pós-graduados, mas isto só ocorre, no momento, em nível de mestrado. É insignificante a presença de doutores nas empresas, revelando, por um lado, o pouco interesse do setor por profissionais mais qualificados ou, por outro, que o profissional qualificado neste nível não tem perfil compatível com as necessidades do setor. Certamente, há uma lacuna entre a academia e a empresa, que necessita urgentemente ser transposta. Por sua vez, constata-se que os Institutos de Pesquisa e empresas que fazem inovação tecnológica (IT) formam seus quadros de recursos humanos à margem dos cursos de Pós-graduação. Dois exemplos significativos são a ITAUTEC e a EMBRAER.

Três novos desafios são de grande relevância nesse contexto: estimular o empreendedorismo e promover a "promiscuidade e a poligamia" intelectual, buscando uma aproximação pró-ativa com o setor empresarial. Logo, o terceiro e quarto eixos mobilizadores recomendados são: Estímulo ao empreendedorismo e à interdisciplinaridade e Aproximação pró-ativa da academia com a atividade econômica. Algumas ações relacionadas a estes eixos podem ser: incentivo e reconhecimento aos programas interdisciplinares e com pesquisa de fronteira; incentivo à busca pelo conhecimento complementar da Química; realização de reforma curricular incluindo atividades que estimulem o empreendedorismo; realização de seminários conjuntos com o setor industrial buscando implementar ligações mais eficientes.

\section{O FINANCIAMENTO À PESQUISA}

Os dois fatos mais importantes para o desenvolvimento da pósgraduação em Química, no Brasil, ocorreram no início dos anos 60 e dos anos 80. O primeiro foi a criação do Funtec, no Banco Nacional de Desenvolvimento Econômico (hoje BNDES), quando este passou a subvencionar programas de PG e atividades de pesquisa fundamental. O segundo, em 1984, foi a criação do PADCT, que definiu a Química e a Engenharia Química como áreas prioritárias.

A partir de 1998, o modelo de financiamento da pesquisa científica e tecnológica pelo Governo Federal passou por mudanças significativas com a introdução dos Fundos Setoriais, Institutos do Milênio, Programas Temáticos do $\mathrm{CNPq}$ e Editais Universais do CNPq. Neste novo cenário, o denominado "balcão" das Agências Federais praticamente desapareceu e, por exemplo, o Edital de Auxílio Individual do $\mathrm{CNPq} \mathrm{N}^{\circ}$ 001/2000, que envolvia recursos totais da ordem de $\mathrm{R} \$ 15$ milhões de reais, recebeu uma demanda de 7.331 projetos, correspondendo a um valor superior a $\mathrm{R} \$ 266$ milhões de reais, para o qual a área de Química apresentou 573 projetos, correspondendo a quase $\mathrm{R} \$ 21$ milhões de reais, ou seja, uma demanda significativamente maior do que o total de recursos disponíveis no Edital. Em 2002, os recursos federais para a pesquisa em Química praticamente desapareceram, ficando restritos à manutenção das bolsas de formação e de pesquisa e ao Portal de periódicos da CAPES (www.periodicos.capes.gov.br). Nos Estados em que existem Fundações de Apoio à Pesquisa, instaladas e atuantes, a situação foi minimizada mas, nos demais, a situação de financiamento está insustentável com grupos de pesquisa sem recursos para o custeio de qualquer espécie.

Uma das razões que levaram a área de Química a apresentar maiores taxas de crescimento na formação de mestres e doutores, bem como na publicação de artigos em periódicos científicos qualificados foi o apoio significativo do Subprograma de Química e Engenharia Química do Programa de Apoio ao Desenvolvimento Científico e Tecnológico - PADCT, na segunda metade da década de 80 e primeira da década de 90 . Naquele período foram apoiados 449 projetos no valor total de US\$ 83 milhões de dólares americanos ${ }^{14,15}$. Os projetos envolveram o apoio a: Formação de RH (Graduação e Pós-Graduação); Grupos de Pesquisa (Consolidados e em Consolidação); Infraestrutura (Centrais Analíticas, Equipamentos Multi-usuários, Bibliotecas e Manutenção); Pesquisa Tecnológica (Projetos Universidade Empresa); Divulgação Científica e a Editoração de Livros Didáticos ${ }^{16}$.

Independentemente do parâmetro considerado, pode-se observar que as taxas de crescimento da área de Química mudam de linear para exponencial a partir dos anos 90; um exemplo disso está representado na Figura 8, que mostra a evolução das teses de doutorado defendidas e o número de publicações em periódicos no exterior. O PADCT reconfigurou a Química no país. O desafio atual é a garantia de recursos financeiros - ampliados em volume, continuados, diversificados e regionalmente sustentados. Logo, o quinto eixo mobilizador sugerido é: Vinculação orçamentária de recursos para C\&T. Algumas ações relacionadas a este eixo podem ser: propor vinculação orçamentária dos recursos para investimento em C\&T, sugerindo um valor inicial de $1 \%$ do PIB, visando atingir $2 \%$ em 10 anos; propor ao Governo Federal que cobre dos governos estaduais uma maior facilidade na execução real dos recursos efetivamente vinculados nas Constituições Estaduais; exigir que os recursos previstos nos Fundos Setoriais sejam 


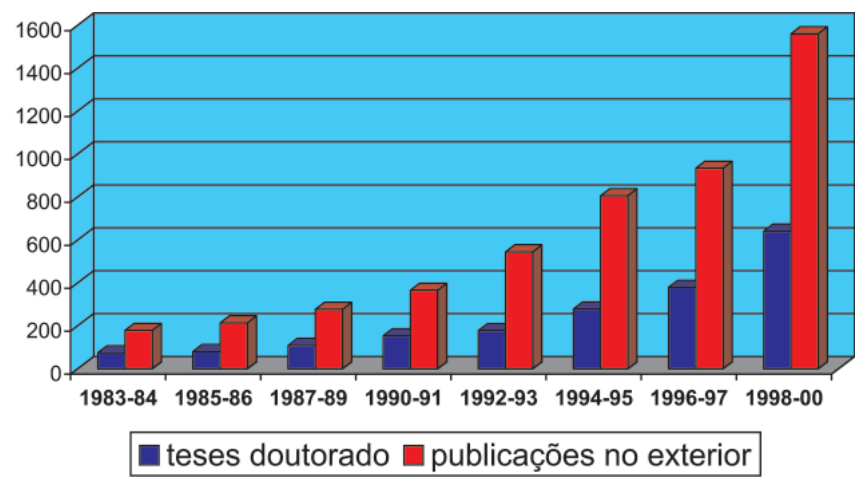

Figura 8. Evolução das teses de doutorado defendidas em Química e o número de publicações em periódicos no exterior

efetivamente colocados nas propostas orçamentárias e fielmente executados; propor a ampliação da participação do CNPq na execução dos Fundos Setoriais; propor que o fundo de Infra-estrutura elabore um conjunto de editais específicos que tratem da Infra-estrutura de Grandes Equipamentos, Infra-estrutura de Acervo Bibliográfico e manutenção de equipamentos científicos; propor que os recursos disponíveis sejam prioritariamente destinados a garantir a continuidade e o crescimento para todos os programas institucionais e/ou individuais de fomento. Com isso, visa-se fortalecer os programas de: Bolsas (aumento de número em todos os casos), Projetos Individuais/Integrados, Taxas de Bancada, Apoio a Jovens Pesquisadores e Grupos Isolados, Programa de Incentivo a cursos de pós-graduação com conceitos 3 e 4; propor o apoio a Redes Temáticas e Redes Interdisciplinares, com financiamento adicional àquele necessário para manutenção do item anterior; propor a criação de um Programa Emergencial de Infra-estrutura, que permita recuperar/montar infra-estrutura de novos e grandes equipamentos.

\section{A SITUAÇÃO INSTITUCIONAL}

A atividade de pesquisa em Química no Brasil é desenvolvida majoritariamente nas universidades públicas. Constata-se, todavia, que a estrutura organizacional dessas instituições apresenta vários gargalos que dificultam o desenvolvimento da pesquisa, dentre os quais destacam-se, entre outros, a estrutura Departamental das IES que se tornou barreira para a interdisciplinaridade e fonte de duplicação de esforços; o Sistema de gestão das IES que é desarticulado, lento e dispendioso; a qualificação e a gestão de pessoal técnico que é inadequada para a atividade de pesquisa; o serviço de manutenção de equipamentos, que é inexistente ou deficiente e, a interlocução com o setor industrial, que é deficiente. Além dos gargalos intrainstitucionais já apontados, precisam ser iniciadas ações visando solucionar problemas relacionados com a importação de equipamentos científicos - que envolvem a INFRAERO - e questões legais relacionadas com seguro - em especial a importação de insumos químicos -, que sofreu um grande estrangulamento com a extinção do SARDI, no contexto da atual conjuntura internacional após o "11 de setembro de 2001" e a possibilidade do recrudescimento de conflitos armados. Nesse sentido, o sexto eixo mobilizador sugerido é: Combate aos gargalos institucionais.

\section{AÇÕES ANTERIORES DA SBQ}

Nestes 25 anos de existência, a Sociedade Brasileira de Química tem sido o principal órgão aglutinador e mobilizador da Química brasileira. Em vários momentos produziu documentos que registra- ram a situação da Química na época e as perspectivas da comunidade. Alguns exemplos significativos dessas ações estão registrados em Química Nova e representam marcos para a Química. Entre outros, podem ser destacados:

i) $\mathrm{Na} 16^{\mathrm{a}}$ Assembléia Geral da Sociedade Brasileira de Química (1993) foi constituída uma comissão composta por Jailson B. de Andrade (UFBA), Heloisa H. R. Schor (UFMG), Marco A. C. do Nascimento (UFRJ), Marcelo Giordan (UNICAMP), Timothy J. Brocksom (UFSCAR/CAPES), Faruk Nome (UFSC) e Eliezer J. Barreiro (UFRJ), para dar continuidade aos trabalhos sobre a "Análise da Pós-Graduação em Química no País”. Dentre os principais resultados, o trabalho realizado pela comissão indicou uma necessidade de investimento da ordem de US\$ 150 milhões de dólares americanos, em cinco anos, que dotariam os cursos de PG em Química de uma boa infraestrutura instrumental; sugeria que o sistema de bibliotecas precisava ser fortalecido; recomendava que a capacitação de estudantes para o magistério fosse considerada fundamental; revelava que a projeção número atual $=>$ número desejável de docentes indicava uma necessidade de incremento médio de $33 \%$ no número de professores da época; e definia a filosofia a ser utilizada na formação de mestres e de doutores ${ }^{17}$;

ii) Em 1995, a Diretoria e Conselho e o Consultivo da SBQ elaboraram o documento "A contribuição da Química ao desenvolvimento científico e tecnológico do país e a necessidade de investimentos", que foi apresentado ao Ministro de Ciência e Tecnologia, ao Presidente do CNPq e à Presidência da Comissão de C\&T da Câmara dos Deputados ${ }^{18}$. Entre outros, o documento revelava a necessidade do valor global de investimentos em recursos estimados para a área, em quatro anos, US\$ 336 milhões de dólares americanos e

iii) A SBQ promoveu uma reunião em Salvador - BA, em agosto de 1996, envolvendo os coordenadores de cursos de pós-graduação em Química, membros da Diretoria e do Conselho da SBQ e membros dos Comitês Assessores de Química da CAPES e do CNPq. A pós-graduação em Química e o fomento à pesquisa foram assuntos amplamente discutidos nessa reunião, parte significativa da qual está registrada em Química Nova ${ }^{19}$.

Uma grande parte das projeções feitas nos documentos anteriores ainda pode ser aplicada à situação atual, podendo-se destacar:

i) Necessidade de um investimento da ordem de US\$ 80 milhões de dólares americanos em quatro anos, nos cursos de graduação de Química e Engenharia Química, para recuperar e ampliar a infraestrutura laboratorial, instrumental e de bibliotecas, melhorar a qualificação profissional dos professores universitários e do segundo grau, além de promover um estímulo à produção de livros e textos especializados em Química, com quantidade e qualidade compatíveis com as necessidades do país;

ii) Necessidade de um investimento da ordem de US\$150 milhões de dólares americanos em quatro anos, nos cursos de pós-graduação em Química, no sentido de modernizar e ampliar a infraestrutura instrumental, de insumos e o gerenciamento e tratamento de resíduos químicos. Vale ressaltar que os investimentos nos cursos de pós-graduação sempre refletem positivamente na graduação;

iii) A criação, pelo CNPq/CAPES, de um programa tipo "enxoval" que poderia consistir em auxílios da ordem de US\$50 mil dóla- 
res americanos, por dois anos, para apoiar a instalação de novos doutores recém-contratados por departamentos de Química. Fazendo uma projeção com o número de doutores formados por ano, isto significaria um investimento, em quatro anos, de cerca de US\$ 26 milhões de dólares americanos;

iv) A vinculação das bolsas de produtividade em pesquisa do $\mathrm{CNPq}$ com recursos anuais variáveis entre US\$ 15 mil e US\$ 25 mil dólares americanos. O valor seria estabelecido em função do nível do bolsista. Isso representaria um valor estimado, em quatro anos, da ordem de US\$ 30 milhões de dólares americanos e

v) Adicionalmente às estimativas anteriores, recursos da ordem de US\$ 50 milhões de dólares americanos, em quatro anos, para a manutenção e a expansão da capacidade instalada de pesquisa em Química.

\section{CONSIDERAÇÕES FINAIS}

Na segunda metade do século XX, a pesquisa científica no Brasil deu um salto significativo. Vários fatores contribuíram para isso, merecendo destaque a criação do CNPq e da CAPES na década de 50 e, posteriormente, da FINEP e FAPESP, bem como a institucionalização e avaliação da pós-graduação. Atualmente, a ciência brasileira está diante de uma mudança de paradigma ${ }^{20}$ : se na década de 60 bastava ao professor universitário fazer pesquisa, na década de 80 a publicação de papers era condição obrigatória para ser considerado pesquisador. Várias vezes, nas últimas cinco décadas, o desafio (perguntas) apresentado aos professores pesquisadores mudou (Figura 9). Provavelmente, em breve, a "pergunta da vez será": quantos empregos a sua pesquisa gerou? Nesse momento próximo, a sintonia da pesquisa científica e tecnológica com a sociedade será inevitável. No caso específico da área de Química, por ser um ramo da ciência a serviço da humanidade, a sintonia é indissociável. A Sociedade Brasileira de Química, mobilizada e motivada espera, com os Eixos Mobilizadores em Química, contribuir nas mudanças efetivas que potencializem o ensino e a geração, difusão e apropriação do conhecimento.

\section{Evolução das \\ Perguntas aos \\ Professores Cientistas}

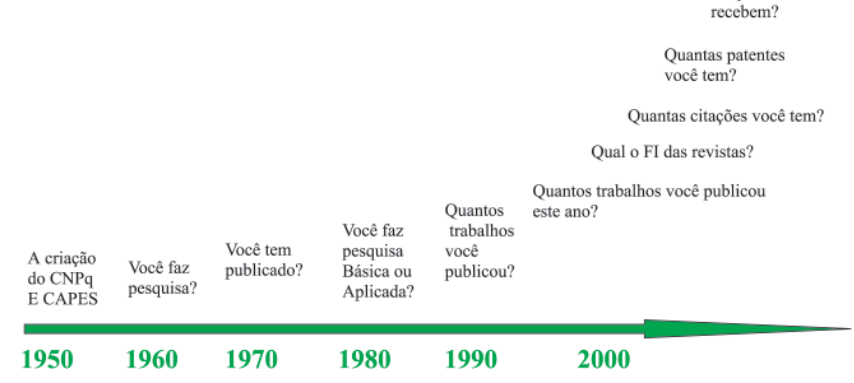

Figura 9. A evolução do paradigma: perguntas feitas aos professores cientistas, adaptado da ref. 20

Não obstante o caráter subjetivo que nossas projeções possam ter, entendemos que este artigo cumprirá integralmente seu objetivo se for motivo de discussão pelo Brasil afora. Não basta que seja apenas do conhecimento dos coordenadores de pós-graduação e dos dirigentes das instituições; é preciso, mais do que nunca, que seja discutido pelos estudantes de Química, notadamente pelos pósgraduandos, para que a área de Química cintinue crescendo, mas que o faça de forma eficaz e, em consonância com as necessidades atuais da sociedade.

\section{AGRADECIMENTOS}

Ao apoio financeiro da CAPES, CNPq e FINEP para a realização dos simpósios e workshop, a Nei Carvalho Bahia e Maria Joana Zucco pelo auxílio na confecção das figuras e aos(às) colegas que participaram das discussões nos workshop realizados: Alfredo Arnóbio Gama; Aldo J. G. Zarbin; A. Celso Spínola Costa; Antonio Luíz Braga; Antonio S. Mangrich; Benedito dos S. Lima Neto; Carlos A. L. Filgueiras; Carlos A. B. Garcia; Cássia C. Turci; Celio Pasquini; Claudia M. de Rezende; Claudio da C. Silveira; Cristina M. A. L. T. M. H. Quintella; Dorila P. Veloso; Eduardo Hollauer; Edvani C. Muniz; Eliezer J. Barreiro; Ernani A. Basso; Faruk Nome; Fernanda R. Garcez; Fernando Galembeck; Francisco C. Nart; Frederico G. Cruz; Germano Tremiliosi Filho; Gil V. J. da Silva; Gilberto Maia; Graciela Arbilla; Hans Viertler; Heloysa M. C. Andrade; Hubert Stassen; Isabel M. N. da S. Moreira; Jari N. Cardoso; João Batista N. da Costa; Joel C. Rubim; Jorge M. David; José D. Fabris; José R. S. Jr.; Judith P. de A. Feitosa; Keiko Takashima; Ledjane S. Barreto; Lucia H. Mascaro; Lucio Angnes; Luis H. M. da Silva; Mara E. F. Braibante; Maria de Fátima D. Costa; Maria do Carmo H. da Silva; Maria Helena Sarragiotto; Maria Rose J. Albuquerque; Mariana H. Chaves; Marília O. F. Goulart; Mauro M. Braga; Maysa Furlan; Munir Skaf; Nelson R. Antoniosi Filho; Oswaldo L. Alves; Otilia D. J. Pessoa; Loyola P. Cavalcanti; Ricardo A. Rebelo; Ricardo M. Kuster; Ridvan N. Fernandes; Rosa Maria S. Maior; Rosângela de A. Epifânio; Sergio L. Cardoso; Susana I. Córdoba de Torresi; Timothy J. Brocksom; Vanderlan da S. Bolzani; Valdir Soldi; Vera L. Pardini; Vitor F. Ferreira; Wagner J. Barreto e Wilson R. Cunha.

\section{REFERÊNCIAS}

1. Marin, J. M.; J. Chem. Educ. 2000, 73, 217.

2. Stokes, D. E.; Pasteurs Quadrant: Basic Science and Technological Innovation, Book News, Inc., Portland: OR All, 1999.

3. Medeiros, L. A.; Pesquisa Fapesp 2002, 72, 14.

4. Pesquisa Fapesp 2001, 67, 14.

5. Sebastian, J.; Infocapes 2001, 9, 7.

6. Peaff, G.; Chem. Eng, News 1996, 26, 15.

7. Tullo, A. H.; Chem. Eng, News 2001, 17, 31.

8. Pinto, A. C.; de Andrade, J. B.; Quim. Nova 1999, 22, 448.

9. Izique, C.; Pesquisa Fapesp 2002, 81, 22.

10. Samios, D.; Infocapes 2001, 9, 66.

11. Pearce, E. M.; Chem. Eng. News 2002, 80, 2.

12. Zucco, C.; Pessine, F. B. T.; de Andrade, J. B.; Quim. Nova 1999, 22, 454

13. Braga, M. M.; Azevedo, S.; Quim. Nova 2002, 25, 696.

14. Castro, L. A. B.; Prescott, E.; Quim. Nova 1997, 20 (Num. Especial), 15.

15. Paniago, E. B.; Quim. Nova 1997, 20 (Num. Especial), 23.

16. Pinto, A. C.; Peixoto, E. M. A.; Galembeck, F.; de Andrade, J. B.; De Paoli, M. A.; Avaca, L. A.; Alves, O. L.; Quim. Nova 1997, 20 (Num. Especial), 81.

17. de Andrade, J. B.; Schor, H. R.; Nascimento, M. A.; Giordan, M. S.; Brocksom, T. J.; Aguilera, F. N.; Barreiro, E. J.; Quim. Nova 1995, 18 , 97.

18. Sociedade Brasileira de Química; Quim. Nova 1995, 18, 509.

19. Brocksom, T. J.; de Andrade, J. B.; Quim. Nova 1997, 20 (Num. Especial), 29.

20. Vercesi, A.; Hogan, D. J.; Chambouleyon, I.; Martinez, J. M.; Arruda, J. R. F.; Alves, O. L.; Arruda, P.; Hoffmann, R.; Fórum de Reflexão Universitária, CADERNO TEMÁTICO, Suplemento do Jornal da UNICAMP, 2002, Ano I, No 12. 